\title{
Emerging Microscopy for Quantum Information Sciences
}

Qiao Qiao ${ }^{1}$, Lijun $\mathrm{Wu}^{1}$, Jun $\mathrm{Li}^{1}$, Shaobo Cheng ${ }^{1}$, Myung-Geun Han ${ }^{1}$, Joseph A. Garlow ${ }^{1}$, Shawn D. Pollard $^{2}$, Xuewen $\mathrm{Fu}^{1}$, Jing Tao ${ }^{1}$, Joseph Wall ${ }^{1}$, and Yimei Zhu ${ }^{*}$

${ }^{1}$ Brookhaven National Laboratory, Upton, NY, USA.

${ }^{2}$ National University of Singapore, Singapore, Singapore.

* Corresponding author: zhu@bnl.gov

Transformative developments in quantum materials and quantum information sciences require knowledge of quantum states of electrons and spins and their competing degrees of freedom. Currently there is an urgent need using electrons as a probe, taking their advantage of strong scattering power with matter, to directly measure intriguing structural behavior and response of quantum materials under external stimuli, such as electric field/potential, resonant spin current, photon pulses and temperature. By developing timeand-space-resolved quantitative emerging microscopy methods we can better understand the role of symmetry, topology, dimensionality, and the interplay of charge-spin-orbital-lattice that control exotic physical properties of materials. It will also help us identify the potential impact of quantum-inspired technologies.

In the past two decades, our group has been developing various approaches to study the correlations of charge, spin, orbital and lattice in strongly correlated electron systems and quantum materials such as superconductors and multiferroics. The approaches include atomically resolved imaging, diffraction, spectroscopy and in-situ magnetoelectric biasing as well as femtosecond ultrafast electron diffraction. In this presentation we will focus on a few case studies. For instance, low-temperature study (down to 10K) of atomic resolution STEM and EELS of single-, or few-unitcell FeSe grown on $\mathrm{SrTiO}_{3}$ substrate to understand the interface enhanced superconductivity (Fig.1) [1]. Investigation of topological state and symmetry breaking of ferroelectric vortices in manganites with and without applied field (Fig.2) [2]. Direct observations and contrast mechanism of Néel Skyrmion in multilayer Co/Pd with strong interfacial Dzyaloshinskii-Moriya interactions [3]. In addition, we report our recent work on 4DSTEM using our newly installed Medipix detector. In Fig.2(f) we show the atomic resolution STEM HAADF images of monolayer $1 \mathrm{H}-\mathrm{MoS}_{2}$, besides observing the adatom at the Mo site we reveal the related charge and electric field, as shown in the projected momentum transfer vector map (Fig.2(g)) and amplitude map (Fig.2(h)). Finally, we give a historical view of the pixelated direct-detector development at BNL [4-7] and the associated analytical approach and structural reconstruction using full 4DSTEM datasets. Our detector development and preliminary work (Fig.2(a-e)) were well documented in the original DOE-BES TEAM project proposal and presented at the TEAM workshops [8].

References:

[1] W. Zhao, et al., Science Advances, 4 (2018), p. eaao2682.

[2] S. Cheng, et al., Phys. Rev. Lett., 118 (2017), p. 145501.

[3] S.D. Pollard, et al., Nature Communication, 8 (2017), p. 14761 | DOI: 10.1038/ncomms14761.

[4] W. Chen, et al., IEEE Transactions on Nuclear Sciences, 49 (2002), p. 1006.

[5] W. Chen, et al., Nuclear Instruments \& Methods in Physics Research A, 512 (2003), p. 368.

[6] P. Rehak, J. Wall and Y. Zhu, Microsc Microanal 11(Suppl 2) (2005), p. 470.

[7] Deptuch, G. et al., Ultramicroscopy 107 (2007), p. 674.

[8] Work at BNL was supported by the US DOE-BES, MSED, under Contract No. DESC0012704. 


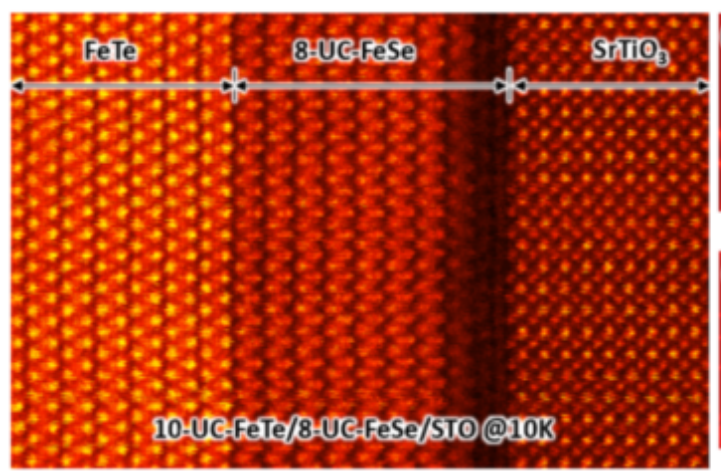

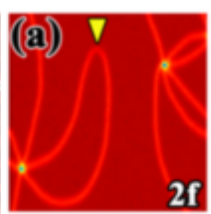

$b=1,[0 \times 1]$

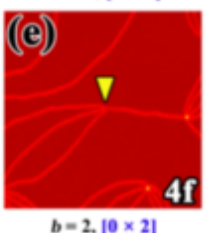

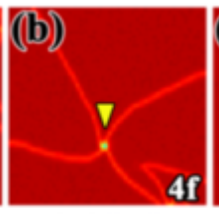

$b=1,[1 \times 1]$

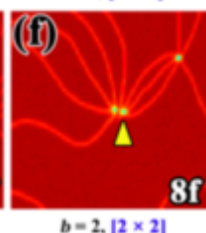

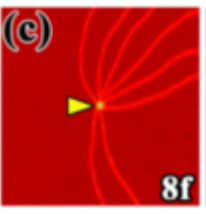

$b=1, \mid-1 \times 1\}$

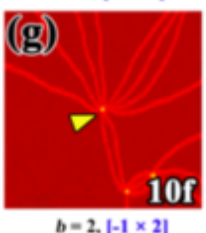

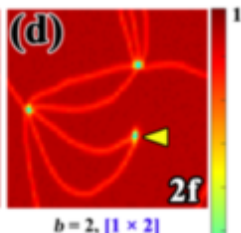

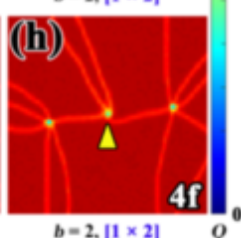

Figure 1. (left) Atom resolution STEM image (false color) acquired at 10K of the 8-unitcell (UC) FeSe grown on $\mathrm{SrTiO}_{3}$ substrate with a 10-UC capping layer of FeTe to study interface enhanced superconductivity [1] using a costume-design liquid-He-temperature biasing holder in cooperation with Gatan-UK/Oxford Instrument. (right) Non-6-fold ferroelectric topological vortices in 6-fold $\mathrm{YMnO}_{3}$ multiferroic oxide based on Landau free energy numerical simulations [2]. All these crystallographically forbidden topologically allowed defects are observed using atomic resolution imaging [2]. The yellow arrows indicate the position of vortex cores and the associated partial dislocations. Corresponding Burgers vectors $\mathrm{b}$ and fundamental homotopy group elements $[\mathrm{m} \times \mathrm{n}]$ (winding numbers) that allow us to classify the topological vortices are given at the bottom of each figure. Color of the figures corresponds to the tilt amplitude $\mathrm{Q}$ of the $\mathrm{MnO}_{5}$ bi-pyramid that gives rise to the topological order and ferroelectric domains in the system. The color legend is shown on the right with the unit of $\AA$.
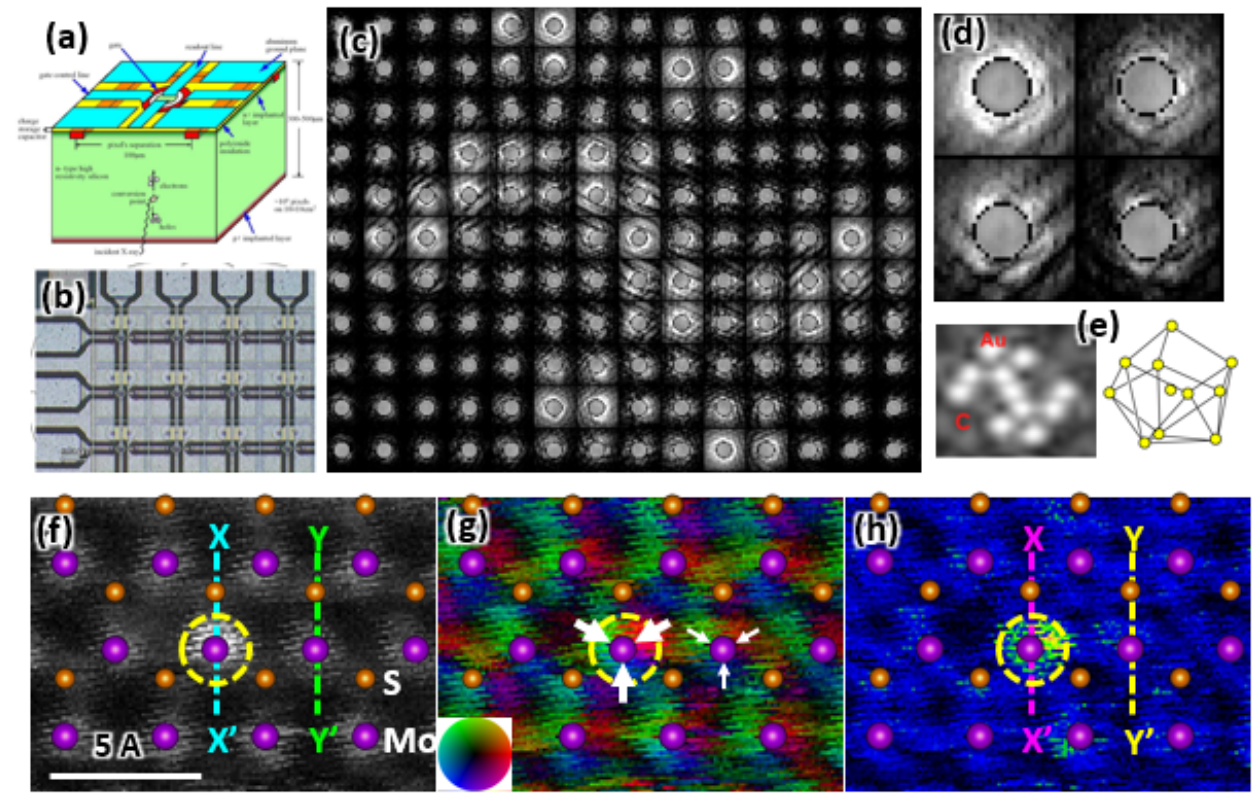

Figure 2. (a-b) A schematic diagram of the pixel structure and a micrograph of several actual pixels of X-ray Active Matrix Pixel Sensor (XAMPS) developed at BNL in the beginning of the new millennium [4-7]. (c-d) Scanning diffraction patterns (4DSTEM) of the cluster of 11 gold atoms. (e) Reconstructed image of the gold cluster using diffuse scattering and its structure model (courtesy of J. Wall of BNL). The direct detector development and 4DSTEM research were part of the original DOE-BES TEAM project and Fig.2(a-e) was documented in the TEAM proposal and presented at TEAM workshops. (f-g) Recent work on 4DSTEM at BNL using the newly installed Medipix detector (Quantum Detectors), showing atomic resolution HAADF image of $\mathrm{MoS}_{2}$ with Mo adatom indicated by the yellow circle in (f). (g) Projected momentum transfer vector map of the same area of (f). White arrows indicate the anisotropy of the vector. (h) Projected momentum transfer magnitude map. Dashed lines in (f) and (h) are intensity scan profiles (not shown here). 